\title{
Impact of a Multimodal Antimicrobial Stewardship Program on Pseudomonas aeruginosa Susceptibility and Antimicrobial Use in the Intensive Care Unit Setting
}

\author{
Douglas Slain, ${ }^{1,2}$ Arif R. Sarwari, ${ }^{1}$ Karen O. Petros, ${ }^{3}$ Richard L. McKnight, ${ }^{3}$ \\ Renee B. Sager, ${ }^{3}$ Charles J. Mullett, ${ }^{4}$ Alison Wilson, ${ }^{5}$ John G. Thomas, ${ }^{6}$ Kathryn Moffett, ${ }^{4}$ \\ H. Carlton Palmer, ${ }^{7}$ and Harakh V. Dedhia ${ }^{8}$ \\ ${ }^{1}$ Division of Infectious Diseases, West Virginia University (WVU), Morgantown, WV 26506-9163, USA \\ ${ }^{2}$ School of Pharmacy, West Virginia University (WVU), Morgantown, WV 26506-9520, USA \\ ${ }^{3}$ Pharmacy Services, WVU Hospitals, Morgantown, WV 26506-8045, USA \\ ${ }^{4}$ Division of Pediatrics, West Virginia University (WVU), Morgantown, WV 26506-9214, USA \\ ${ }^{5}$ Division of Surgery, West Virginia University (WVU), Morgantown, WV 26506-9238, USA \\ ${ }^{6}$ Department of Pathology, West Virginia University (WVU), Morgantown, WV 26506-9203, USA \\ ${ }^{7}$ Department of Internal Medicine, West Virginia University (WVU), Morgantown, WV 26506-9160, USA \\ ${ }^{8}$ Division of Pulmonary-Critical Care Medicine, West Virginia University (WVU), Morgantown, WV 26506-9166, USA
}

Correspondence should be addressed to Douglas Slain, dslain@hsc.wvu.edu

Received 10 December 2010; Revised 15 March 2011; Accepted 15 March 2011

Academic Editor: Roland M. Schein

Copyright () 2011 Douglas Slain et al. This is an open access article distributed under the Creative Commons Attribution License, which permits unrestricted use, distribution, and reproduction in any medium, provided the original work is properly cited.

\begin{abstract}
Objective. To study the impact of our multimodal antibiotic stewardship program on Pseudomonas aeruginosa susceptibility and antibiotic use in the intensive care unit (ICU) setting. Methods. Our stewardship program employed the key tenants of published antimicrobial stewardship guidelines. These included prospective audits with intervention and feedback, formulary restriction with preauthorization, educational conferences, guidelines for use, antimicrobial cycling, and de-escalation of therapy. ICU antibiotic use was measured and expressed as defined daily doses (DDD) per 1,000 patient-days. Results. Certain temporal relationships between antibiotic use and ICU resistance patterns appeared to be affected by our antibiotic stewardship program. In particular, the ICU use of intravenous ciprofloxacin and ceftazidime declined from 148 and $62.5 \mathrm{DDD} / 1,000$ patient-days to 40.0 and 24.5 , respectively, during 2004 to 2007. An increase in the use of these agents and resistance to these agents was witnessed during 20082010. Despite variability in antibiotic usage from the stewardship efforts, we were overall unable to show statistical relationships with $P$. aeruginosa resistance rate. Conclusion. Antibiotic resistance in the ICU setting is complex. Multimodal stewardship efforts attempt to prevent resistance, but such programs clearly have their limits.
\end{abstract}

\section{Introduction}

Intensive care units (ICUs) have the highest density of antimicrobial use and the highest rates of bacterial resistance. Institution-wide and specific unit-based antibiotic stewardship initiatives have been advocated by many as a way to preserve the utility of antimicrobial agents [1]. The Infectious Diseases Society of America (IDSA) and the Society for Healthcare Epidemiology of America (SHEA) have developed guidelines for antibiotic stewardship to help combat bacterial resistance [2]. The two core tenets of these guidelines are use of "prospective audits with intervention and feedback" and "formulary restriction and preauthorization." Additional elements of an antibiotic stewardship program could include: education, guidelines and clinical pathways, antimicrobial cycling, and de-escalation of therapy.

Prior to the release of the IDSA/SHEA guidelines, a multimodal institution-wide antimicrobial stewardship program was developed at our university hospital which incorporated many of the elements listed in the guideline. Adult ICUs 
were especially targeted by our stewardship initiatives due to the high baseline use of antibiotics. A major impetus for developing our program was to improve or preserve Pseudomonas aeruginosa susceptibility given the limited number of antibiotics with activity against this important pathogen. In particular, we had witnessed a jump in $P$. aeruginosa resistance to ciprofloxacin from $38 \%$ in 2003 to $56 \%$ in 2004 in the adult ICUs before the start of the program. The objectives of this study are to describe how implementation of an institutional multimodal stewardship program affected $P$. aeruginosa susceptibility and antibiotic use in the ICU setting.

\section{Material and Methods}

2.1. Hospital and Stewardship Efforts. Our institution is a 531-bed academic medical center. Adult ICUs include a Cardiothoracic Unit, Coronary Care Unit, closed-model Medical Intensive Care Unit (MICU), and a Surgical Intensive Care Unit (SICU). Both the MICU and SICU have dedicated unitbased critical care physicians and clinical pharmacy specialists. In addition, clinical staffing pharmacists verify orders on all shifts and are instructed to intervene on restricted medication orders if used outside of criteria. They also make sure that the teams are following the antibiotic cycling.

During the study period, no significant changes were implemented in terms of infection control practices except for an increase in alcohol-based hand sanitizer use during the 2009-2010 H1N1 influenza pandemic. Wall dispensers were placed at the entrance to each hospital patient's room and at all ICU entrances. This study was conducted in accordance with the Declaration of Helsinki (1964) and was granted exempt status by the University Institutional Review Board.

Our stewardship program incorporates prospective audits with intervention and feedback, formulary restriction and preauthorization, educational noon conferences for physicians and pharmacists, institutional pocket card guidelines, and a ventilator-associated pneumonia (VAP) antimicrobial cycling protocol with streamlining/de-escalation. The stewardship program was implemented in step-wise fashion starting in the fall of November 2004 with the development of a new high-peak aminoglycoside dosing protocol, and antibiotic educational initiatives including conferences. In January of 2005, criteria-based restrictions were placed on broad spectrum antibacterials. In particular, the new criteria restricted all fluoroquinolone use as first-line agents in the ICU. Ciprofloxacin, the ICU fluoroquinolone of choice, was deemed appropriate only in the setting of presumed or documented infections with Pseudomonas aeruginosa in patients allergic or intolerant to $\beta$-lactams or aminoglycosides. In July 2005 , the VAP protocol was initiated as a way to increase the heterogeneity of antibiotic use. The antibiotic selections were broadly based on guidelines published by the American Thoracic Society [3]. Cycling of gram-negative agents (a carbapenem, cefepime, and piperacillin-tazobactam) was performed quarterly whereas cycling of gram-positive agents (vancomycin and linezolid) was performed semiannually. The VAP protocol was also designed to reduce the amount of ciprofloxacin and ceftazidime use given the increased resistance rates at the time. Imipenem was the primary carbapenem used during 2003-2009. A formulary switch to doripenem was made for 2010. Limited use of meropenem was permitted throughout the study period. De-escalation involved a switch to a narrower spectrum agent of the same antibiotic class (imipenem to ertapenem, cefepime to ceftriaxone, and piperacillin-tazobactam to ampicillinsulbactam) at 48-72 hours. De-escalation also involved the discontinuation of the empiric gram-positive agent if MRSA was not isolated. Ceftazidime use was discouraged in favor of cefepime throughout the ICU setting. Finally, in August of 2005, pocket cards with antibiograms and institutional restriction and usage guidelines were distributed to physicians and pharmacists along with educational conferences. This has since become an annual event for incoming house staff. In addition, the pocket card data is available on the institution's intranet website.

Since it was not logistically possible to assess each segment of this multimodal program on antimicrobial resistance in the ICU setting, we decided to focus our assessment on the global use of anti-pseudomonal agents in the adult ICUs and compared the use of these agents with $P$. aeruginosa resistance in the same ICUs.

2.2. ICU Antibiotic Utilization Data. ICU antibiotic usage was collected from patient billing databases for 2003 to 2008 . Billing data is derived from nursing medication administration data. Antibiotic usage was converted from total grams used to defined daily doses (DDD) per 1,000 patient-days to make year-to-year comparisons. The DDD conversion factors were developed from the World Health Organization definitions for individual antibiotics (http://www.whocc .no/atc_ddd_index/). This is one of the most commonly used methods to measure antibiotic consumption $[4,5]$.

2.3. ICU Resistance Determination. Nonduplicative adult ICU specimens from all sources were analyzed in this study. Susceptibility was determined using Vitek and Vitek 2 automated systems (bioMérieux). Carbapenem resistance rates were represented by imipenem resistance in this study since it was the carbapenem agent used the most and since doripenem susceptibility testing was not available with our automated system during the majority of the study. Automated susceptibility testing for piperacillin-tazobactam and cefepime were not performed until 2005 when these agents became formulary agents. Susceptibility results were interpreted according to Clinical Laboratory Standards Institute (CLSI) guidelines. Resistance data was expressed and compared using CLSI "resistant" breakpoints rather than "nonsusceptible" breakpoints. Breakpoint values did not change during this study. Clinical laboratory data were exported to The Surveillance Network (TSN) Database (Focus Technologies, Herndon, VA) for benchmarking. Semi-annual reports were obtained from TSN for analysis.

2.4. Statistical Analysis. Linear correlation analysis was performed to assess possible relationships between antibiotic usage and resistance in $P$. aeruginosa. All data was assessed 
using JMP V9.0 Statistical software (SAS Institute, Inc, Cary NC.). Univariate analysis of the use and resistance rates of the same antibiotic was performed followed by bivariate analysis looking at possible relationships with other antibiotics. $P$ values of $<.05$ were considered statistically significant.

\section{Results}

One of the most compelling findings of our study is that overall antibiotic use decreased in the ICU setting as well as in the whole institution between 2004 and 2007. ICU use of key anti-pseudomonal agents decreased from 412 $\mathrm{DDD} / 1,000$ patient-days in 2004 to $346 \mathrm{DDD} / 1,000$ patientdays in 2007. The proportion of antibiotics in the overall pharmacy medication budget decreased from $15.8 \%$ in 2003 to $8.3 \%$ in 2007 . Antibiotic costs decreased as some agents became generic and overall pharmacy purchase of some expensive biological and cancer agents also increased, thus the antibiotic proportion of the pharmacy budget decreased. Unfortunately, in 2010, the ICU use of anti-pseudomonal agents increased to $456 \mathrm{DDD} / 1,000$ patient-days. This most likely reflected the increased incidence in resistant organisms reported in 2008-2010. Changes in ICU anti-pseudomonal usage patterns between 2003 and 2010 appear to reflect a greater degree of heterogeneity. The proportion of antibiotics used in highest frequency at the beginning of the study period decreased, and the proportion of less frequently used antibiotics increased by the end of the study period. Usage patterns of anti-pseudomonal agents in the adult ICUs are reported in Table 1. Fluctuation in usage appears to be affected by the VAP protocol. Since only three gram-negative agents were used in the VAP protocol on a quarterly cycle, this required that the first and fourth quarters would use the same agent within the year.

Yearly ICU antibiotic resistance in Pseudomonas aeruginosa appears in Figure 1. The most significant change in antimicrobial resistance patterns was observed in the reduction of ciprofloxacin-resistant $P$. aeruginosa from $56.2 \%$ in 2004 to $18.4 \%$ in 2006 . Ceftazidime use decreased throughout the study period. Ciprofloxacin use decreased substantially until 2007 while cefepime, tobramycin, and piperacillintazobactam increased throughout the study. Unfortunately, the ciprofloxacin resistance rate increased to $31.0 \%$ in 2007 and then to $47.6 \%$ in 2010 . This rebound of resistance appears to be consistent with increased use of ciprofloxacin during 2008-2010. However, the resistance to ciprofloxacin was still lower than the preprogram rates. Ceftazidimeresistant $P$. aeruginosa also decreased from $31.2 \%$ in 2004 to $18.4 \%$ in 2006 . Unfortunately, the resistance rate increased to between $16.4 \%$ and $20.7 \%$ since 2007 . Piperacillintazobactam and cefepime were not added to the hospital formulary until the later part of 2003 , so their susceptibilities were not routinely performed until 2005 . Resistance to these two agents appears to have increased as their use has increased since 2005. In general, the resistance rates do not appear to be impacted by any lapses in standard infection control practices as clonal resistance outbreaks were not reported during the study period.

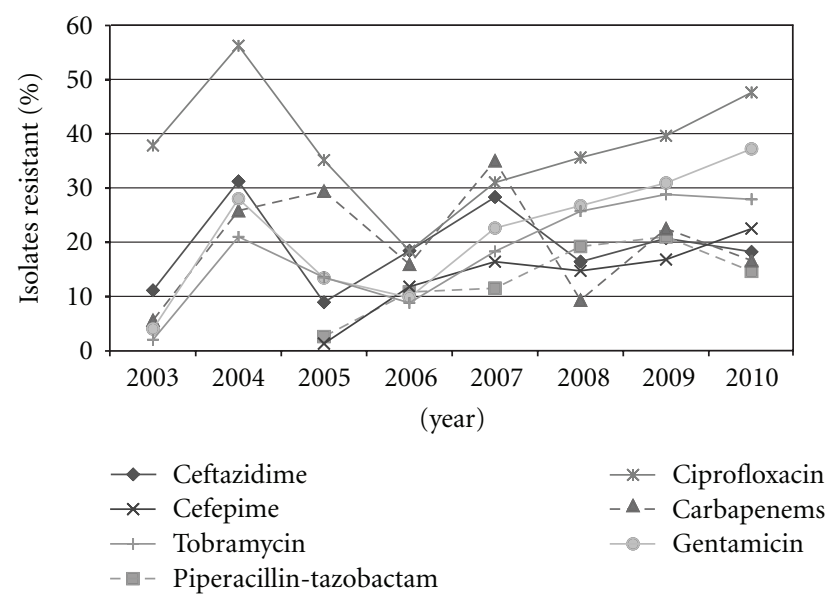

Figure 1: Percent of intensive care unit $P$. aeruginosa isolates resistant to various anti-pseudomonal agents.

Data from the linear correlation analysis are reported in Table 2. In univariate analysis of each antibiotic's usage and resistance, statistical significance was not reached despite the fact that $35 \%$ of the variability in ciprofloxacin resistance and $54 \%$ of variability in cefepime resistance appeared to be associated with the variability in usage of those agents. In bivariate analysis, tobramycin use was inversely correlated with ciprofloxacin resistance and cefepime use was significantly associated with ceftazidime resistance.

\section{Discussion}

The design of this practice-based study was not to specifically measure the impact of any individual initiative with changes in resistance patterns, but rather to look at the impact of a multimodal stewardship program on ICU Pseudomonas susceptibility. A majority of published studies on antibiotic use and resistance have reported increased resistance with increased use of antibiotics [6]. Our study is one in the minority of studies to show a decrease in ciprofloxacin and ceftazidime resistance among ICU $P$. aeruginosa isolates with decreased ciprofloxacin and ceftazidime use. Nguyen and colleagues also recently reported a decrease in ciprofloxacinresistant $P$. aeruginosa in a case-control study; however, their analysis was not limited to the ICU setting [7]. Of course, establishing a direct link between antibiotic use and any degree of attributable resistance is not easily done.

Enforcement of class restrictions can reduce the use of particular antibiotics. A common consequence is that increases in other classes often result in increased resistance to those agents. Such relationships have been referred to as "balloon squeezing" resistance patterns [8]. Antibiotic restrictions at our hospital along with the VAP cycling protocol appeared to affect overall usage patterns in the ICUs. The VAP cycling protocol was specifically designed to improve susceptibility to ciprofloxacin and ceftazidime, as neither was included in the cycling protocol. Cefepime has replaced ceftazidime at many hospitals, but it may not protect against cephalosporin resistance in Pseudomonas. 
TABLE 1: Intensive care unit anti-pseudomonal antibiotic utilization (2003-2010).

\begin{tabular}{lcccccccc}
\hline & 2003 & 2004 & 2005 & 2006 & 2007 & 2008 & 2009 & 2010 \\
\hline Cefepime & 4.5 & 4.0 & 18.5 & 36.5 & $68.5^{\dagger}$ & $\mathbf{9 2 . 5}$ & 36.0 & $\mathbf{9 0 . 5 ^ { \dagger }}$ \\
Ceftazidime & $\mathbf{9 0 . 5}$ & $\mathbf{6 2 . 5}$ & 53.0 & 38.0 & 24.5 & 39.0 & 23.5 & 25.5 \\
Piperacillin-tazobactam & 87.0 & 124.5 & 127.5 & $115.5^{\dagger}$ & 97.5 & $\mathbf{1 3 9 . 0}$ & $130.0^{\dagger}$ & $\mathbf{1 3 3 . 0}$ \\
Carbapenems & 30.0 & 52.5 & 53.0 & 60.0 & 48.5 & $\mathbf{1 1 2 .}^{\dagger}$ & $\mathbf{8 8 . 5}$ & 78.5 \\
Ciprofloxacin & $\mathbf{1 9 2 . 5}$ & $\mathbf{1 4 8 . 0}$ & 57.8 & 25.5 & 40.0 & 66.5 & 48.0 & 87.0 \\
Gentamicin & 10.5 & 20.5 & $\mathbf{4 0 . 0}$ & $\mathbf{2 6 . 5}$ & 8.5 & 17.5 & 10.5 \\
Tobramycin & 1.0 & 0.5 & 42.5 & $\mathbf{5 6 . 5}$ & $\mathbf{5 8 . 0}$ & 48.0 & 35.0 & 31.0 \\
\hline
\end{tabular}

Data expressed as defined daily dose (DDD) per 1000 patient-days.

The two years with the highest percentage of specific antibiotic use are bolded.

tYear with two cycles of this antibiotic in VAP protocol.

TABLE 2: Assessment of correlation between P. aeruginosa resistance and antibiotic usage.

\begin{tabular}{|c|c|c|c|}
\hline Parameters & Correlation coefficient $(r)$ & $r^{2}$ & $P$ value \\
\hline \multicolumn{4}{|l|}{ Univariate assessments } \\
\hline Ciprofloxacin & 0.593 & 0.351 & .121 \\
\hline Ceftazidime & 0.338 & 0.114 & .413 \\
\hline Cefepime & 0.734 & 0.538 & .097 \\
\hline Piperacillin-tazobactam & 0.386 & 0.149 & .450 \\
\hline Carbapenems & 0.219 & 0.048 & .602 \\
\hline Gentamicin & 0.374 & 0.139 & .362 \\
\hline Tobramycin & 0.184 & 0.034 & .664 \\
\hline \multicolumn{4}{|l|}{ Bivariate assessments $^{\dagger}$} \\
\hline Ciprofloxacin resistance & -0.735 & -0.540 & .038 \\
\hline \multicolumn{4}{|l|}{ Tobramycin usage } \\
\hline Ceftazidime resistance & 0.966 & 0.934 & .002 \\
\hline Cefepime use & & & \\
\hline
\end{tabular}

${ }^{\dagger}$ Only relationships with $P$ values $<.05$ are listed.

Not only did we find a trend in the univariate assessment of cefepime use and resistance, but we did see an association with cefepime use and ceftazidime resistance.

We cannot easily explain the increase in $P$. aeruginosa ciprofloxacin resistance in 2007-2010 after the initial reduction in ciprofloxacin usage. However, we did see an increase in the use of ciprofloxacin usage in our ICUs during the first half of 2007 and in 2010. This may explain the $r^{2}$ value of 0.35 . Interestingly, we also witnessed an increase in multipleclass resistance in 2007 and 2010. This may be a reflection of national trends and perhaps a consequence of upregulation of multidrug efflux pumps with use of other agents [9-11]. At least one other group of investigators has postulated the maintenance of multidrug-resistant $P$. aeruginosa during an antibiotic cycling study [12].

The major limitation to our study is the inability to separate out the consequences of each initiative in our multimodal program. In addition, we could not assess any contribution to resistance from horizontal transmission due to lapses in infection control, or as a result of antibiotic use outside of the ICU or in the community. In addition, much smaller amounts of non-pseudomonal antibiotics were used in the ICUs during the time of study and not factored into our analysis. Despite these limitations, we were still able to highlight a few trends by the temporal relationships of the antibiotic usage patterns and the resistance patterns. Our data also suggests that despite interventions such as the VAP protocol and limiting ciprofloxacin and ceftazidime use, it is very difficult to reduce anti-pseudomonal resistance in the ICU setting.

\section{Conclusions}

We are one of the earlier institutions to provide results from an antibiotic stewardship program that uses multiple measures advocated in the IDSA/SHEA guidelines to prevent or delay ICU antimicrobial resistance. Our multimodal program appears to be associated with certain beneficial trends in antibiotic usage and resistance. However, these efforts may not always be able to control natural resistance patterns. More research is required in this area.

\section{Conflict of Interests}

D. Slain has received speaker's honoraria from Wyeth Pharmaceuticals. All other authors report no potential conflicts of interest relevant to this paper. 


\section{Acknowledgments}

The authors wish to thank Dr. Patricia Miller-Canfield and Ms. Debby Tanaguchi, Department of Clinical Laboratories, West Virginia University Hospitals, Mr. Hamed Motlagh for assistance with collection and interpretation of microbiological data, and Gerry Hobbs, Ph.D., associate professor of statistics at west Virginia University for his assistance with statistical analysis. This paper presented at Abstract P-18 2008 National Foundation for Infectious Diseases (NFID) Conference on Antimicrobial Resistance. June 23-35, 2008, Bethesda, Md.

\section{References}

[1] H. Hanberger, D. Arman, H. Gill et al., "Surveillance of microbial resistance in European Intensive Care Units: a first report from the Care-ICU programme for improved infection control," Intensive Care Medicine, vol. 35, no. 1, pp. 91-100, 2009.

[2] T. H. Dellit, R. C. Owens, J. E. McGowan et al., "Infectious Diseases Society of America and the Society for Healthcare Epidemiology of America guidelines for developing an institutional program to enhance antimicrobial stewardship," Clinical Infectious Diseases, vol. 44, no. 2, pp. 159-177, 2007.

[3] M. S. Niederman, D. E. Craven, M. J. Bonten et al., "American Thoracic Society, Infectious Diseases Society of America. Guidelines for the management of adults with hospitalacquired, ventilator-associated, and healthcare-associated pneumonia," American Journal of Respiratory and Critical Care Medicine, vol. 171, pp. 388-416, 2005.

[4] M. Miller, L. Bernard, M. Thompson, D. Grima, and J. Pepin, "Lack of increased colonization with vancomycinresistant enterococci during preferential use of vancomycin for treatment during an outbreak of healthcare-associated clostridium difficile infection," Infection Control and Hospital Epidemiology, vol. 31, no. 7, pp. 710-715, 2010.

[5] P. P. Cook, P. G. Catrou, J. D. Christie, P. D. Young, and R. E. Polk, "Reduction in broad-spectrum antimicrobial use associated with no improvement in hospital antibiogram," Journal of Antimicrobial Chemotherapy, vol. 53, no. 5, pp. 853$859,2004$.

[6] C. R. Friedman and C. G. Whitney, "It's time for a change in practice: reducing antibiotic use can alter antibiotic resistance," Journal of Infectious Diseases, vol. 197, no. 8, pp. 10821083, 2008.

[7] L. H. Nguyen, D. I. Hsu, V. Ganapathy, K. Shriner, and A. Wong-Beringer, "Reducing empirical use of fluoroquinolones for Pseudomonas aeruginosa infections improves outcome," Journal of Antimicrobial Chemotherapy, vol. 61, no. 3, pp. 714720, 2008.

[8] J. J. Rahal, C. Urban, D. Horn et al., "Class restriction of cephalosporin use to control total cephalosporin resistance in nosocomial Klebsiella," Journal of the American Medical Association, vol. 280, no. 14, pp. 1233-1237, 1998.

[9] D. M. Livermore, "Multiple mechanisms of antimicrobial resistance in Pseudomonas aeruginosa: our worst nightmare?" Clinical Infectious Diseases, vol. 34, no. 5, pp. 634-640, 2002.

[10] D. T. Tsukayama, H. J. V. Loon, C. Cartwright et al., "The evolution of Pseudomonas aeruginosa during antibiotic rotation in a medical intensive care unit: the RADAR-trial," International Journal of Antimicrobial Agents, vol. 24, no. 4, pp. 339-345, 2004.
[11] V. H. Tam, K. T. Chang, K. Abdelraouf et al., "Prevalence, resistance mechanisms, and susceptibility of multidrugresistant bloodstream isolates of Pseudomonas aeruginosa," Antimicrobial Agents and Chemotherapy, vol. 54, no. 3, pp. 1160-1164, 2010.

[12] T. L. Hedrick, A. S. Schulman, S. T. Mcelearney et al., "Outbreak of resistant Pseudomonas aeruginosa infections during a quarterly cycling antibiotic regimen," Surgical Infections, vol. 9, no. 2, pp. 139-152, 2008. 


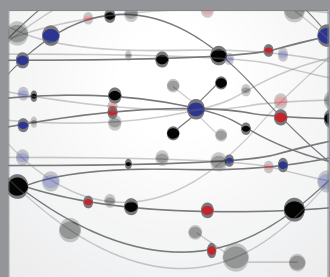

The Scientific World Journal
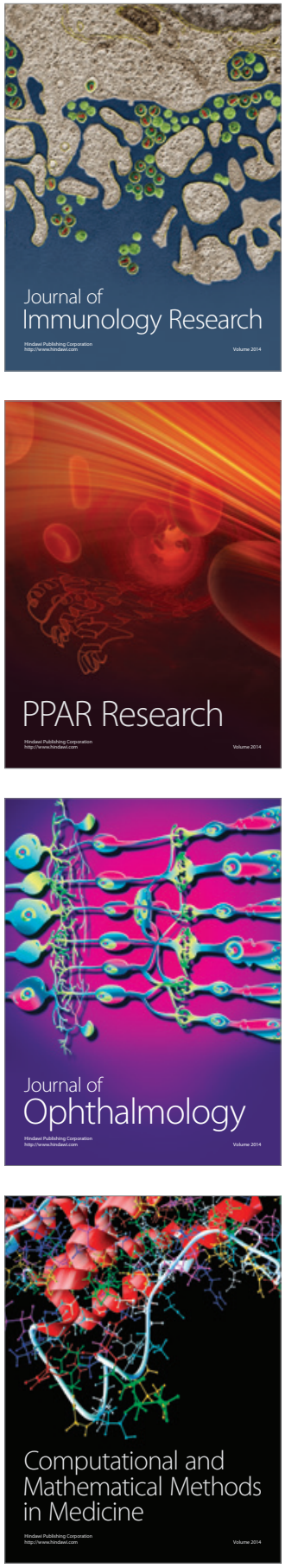

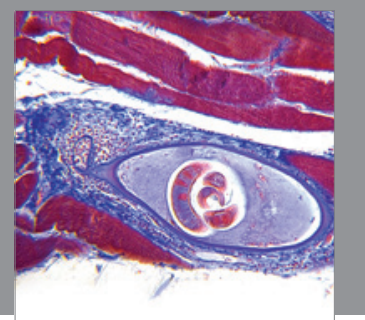

Gastroenterology

Research and Practice
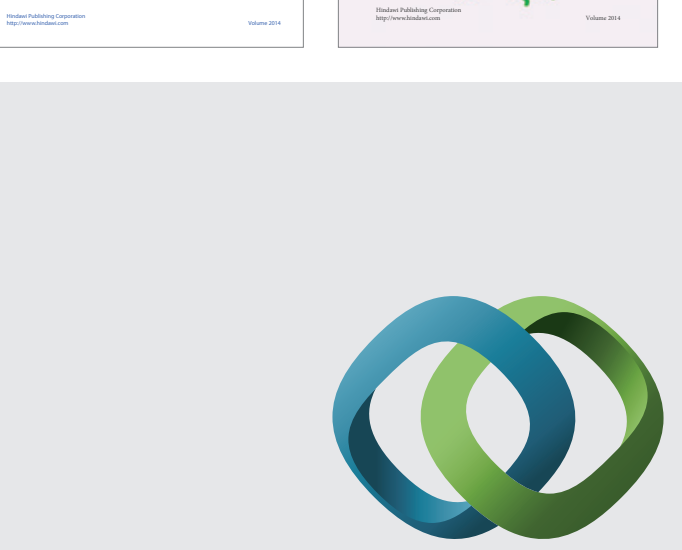

\section{Hindawi}

Submit your manuscripts at

http://www.hindawi.com
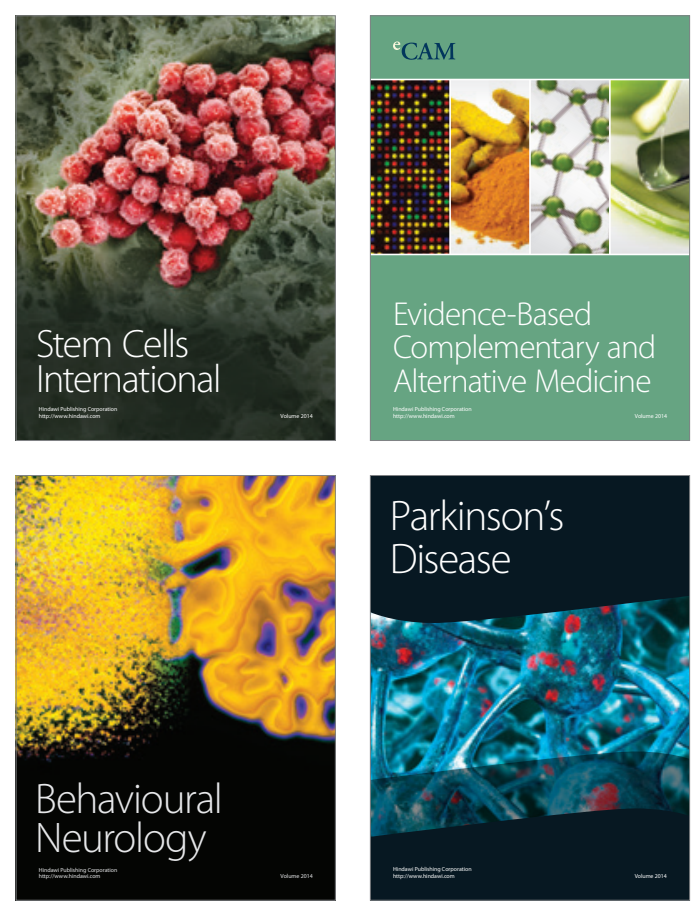

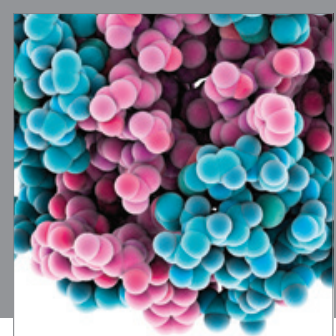

Journal of
Diabetes Research

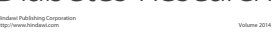

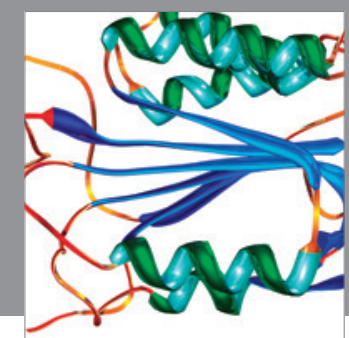

Disease Markers
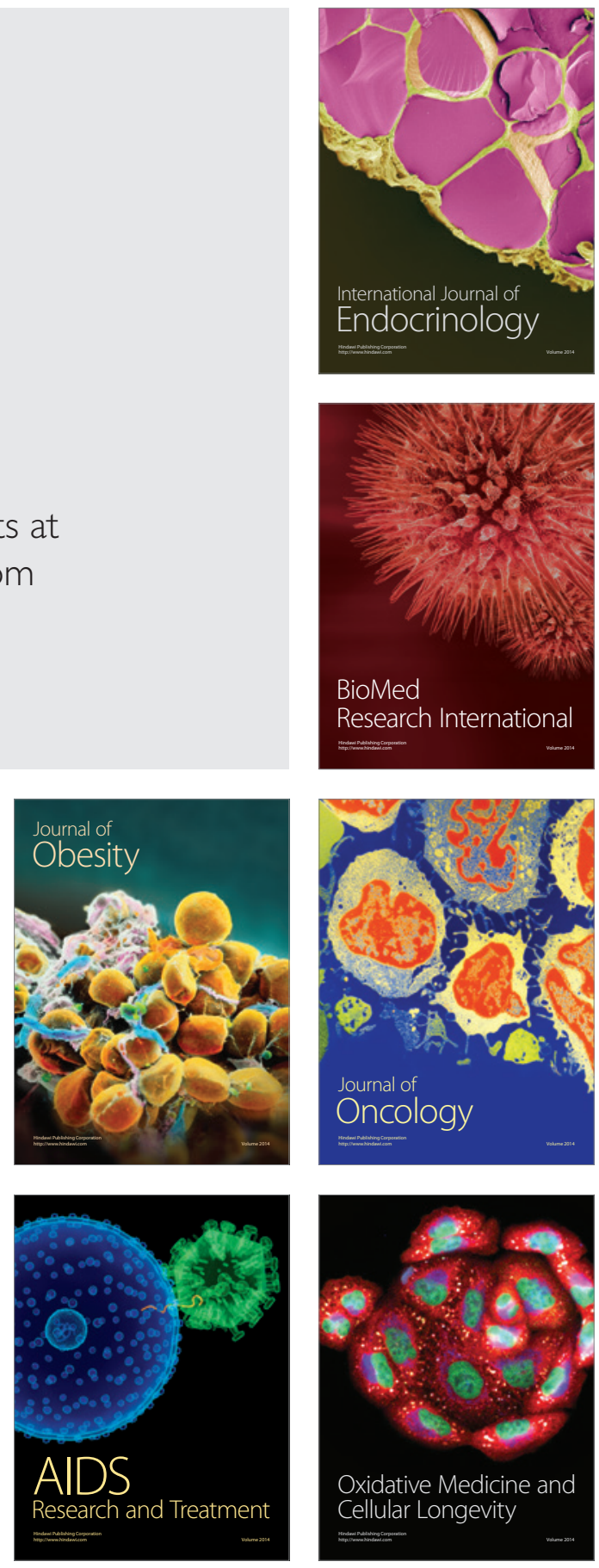